\title{
Effects of Dapagliflozin Compared with Sitagliptin and Metformin in Drug-Naïve Japanese Patients with Type 2 Diabetes: A 12-Week, Open-Label, Randomized, Active-Controlled Trial
}

\author{
Daisuke Ito (D) - Kazuyuki Inoue - Daigo Saito - Keiko Hamaguchi • \\ Kimie Kaneko · Takashi Sumita · Kouichi Inukai · Ikuo Inoue • \\ Akira Shimada
}

Received: September 19, 2021 / Accepted: October 15, 2021 / Published online: October 28, 2021

(C) The Author(s) 2021

\section{ABSTRACT}

Introduction: To compare the efficacy and tolerability of dapagliflozin with those of sitagliptin and metformin in patients with type 2 diabetes who have never received glucose-lowering agents.

Methods: In this randomized, 12-week, openlabel, active-controlled trial, 32 patients were randomly assigned to receive dapagliflozin $5 \mathrm{mg}$, sitagliptin $50 \mathrm{mg}$, or metformin $1000 \mathrm{mg}$ per day for 12 weeks. At baseline and at week 12, the patients underwent a meal tolerance test (MTT).

D. Ito $(\bowtie) \cdot$ K. Inoue $\cdot$ D. Saito $\cdot$ T. Sumita

I. Inoue $\cdot$ A. Shimada

Department of Endocrinology and Diabetes,

Saitama Medical University, 38, Morohongo,

Moroyama, Iruma-gun, Saitama 350-0495, Japan

e-mail: dito@saitama-med.ac.jp

D. Ito $\cdot$ T. Sumita

Department of Internal Medicine, Ogawa Red Cross

Hospital, Saitama, Japan

K. Hamaguchi · K. Kaneko

Department of Nursing, Ogawa Red Cross Hospital,

Saitama, Japan

K. Inukai

Department of Diabetes and Endocrinology,

Higashiyamato Hospital, Tokyo, Japan

I. Inoue

Department of Health Service Center, Saitama

Medical University, Saitama, Japan
Results: After 12 weeks of treatment, the changes in fasting and postprandial plasma glucose and plasma glucose area under the curve (AUC) $0-120$ min levels during the MTT from baseline were significantly improved in the three study groups, and there were no significant differences among the three study groups $(P<0.05)$. The mean changes in glycated hemoglobin (HbA1c) from baseline to week 12 were $-0.96 \%,-1.24 \%$, and $-1.40 \%$ in the dapagliflozin, sitagliptin, and metformin groups, respectively. Although there was no significant difference among the three study groups, the lowering effect of HbA1c tended to be greater in the metformin group than in the dapagliflozin group. In contrast, the insulin

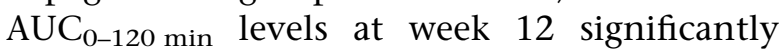
decreased only in the dapagliflozin group $(P=0.049)$. Similarly, body weight was significantly reduced only in the dapagliflozin group $(-2.1 \mathrm{~kg} \quad[-2.7 \%], \quad P=0.047)$. Moreover, dapagliflozin significantly improved serum adiponectin levels $(P=0.003)$. However, there were no significant differences in the changes in these glycemic and metabolic parameters among the three study groups. No serious adverse events were documented in any group. Conclusions: Dapagliflozin exerted beneficial effects similar to sitagliptin and metformin on glycemic parameters. In addition, dapagliflozin significantly reduced body weight and insulin AUC levels and improved serum adiponectin levels. Therefore, we suggest that these three 
hypoglycemic agents could be viable first-line medications for drug-naïve Japanese patients with type 2 diabetes.

Trial Registration: University Hospital Medical Information Network (UMIN000024427).

Keywords: Sodium-glucose cotransporter 2 (SGLT2) inhibitor; Dapagliflozin; Sitagliptin; Metformin; Meal tolerance test; Type 2 diabetes

\section{Key Summary Points}

\section{Why carry out this study?}

Currently, the first-line medications for type 2 diabetes have not been clarified in the guidelines or algorisms in Japan

Sodium-glucose cotransporter 2 (SGLT2) inhibitors have become an early recommended drug for patients with type 2 diabetes at a high risk of cardiovascular events; however, its effectiveness as a firstline medication for Japanese patients with newly diagnosed type 2 diabetes with no history of cardiovascular events is unclear

The aim of the present study was to compare the efficacy of dapagliflozin with those of sitagliptin and metformin, on plasma glucose and insulin levels and related biomarkers during a meal tolerance test in drug-naïve Japanese patients with type 2 diabetes

\section{What was learned from the study?}

After 12 weeks of treatment, dapagliflozin exerted beneficial effects similar to sitagliptin and metformin on glycemic parameters and significantly reduced body weight and insulin levels

These results suggest that along with metformin and dipeptidyl peptidase- 4 (DPP-4) inhibitors, SGLT2 inhibitors could be a viable first-line treatment option for drug-naïve Japanese patients with type 2 diabetes because of their optimum glucose-lowering properties, ability to avoid hypoglycemia or weight gain, and tolerability over a wide range of ages

\section{INTRODUCTION}

In Japan, the estimated number of patients with diabetes is $>10$ million and continues to increase [1]. In previous trials, the United Kingdom Prospective Diabetes Study (UKPDS) clarified the importance of early intensive glucose therapy in patients with newly diagnosed type 2 diabetes to reduce the risk of microvascular complications [2]. The Steno-2 Study and the Japan Diabetes Optimal Integrated Treatment study for three major risk factors of cardiovascular diseases (J-DOIT3) revealed the importance of multifactorial risk reduction, including not only glycemic control but also blood pressure and lipid level reduction and other metabolic management focusing on macrovascular risk mitigation among patients with type 2 diabetes $[3,4]$. However, there is concern that intensive glucose-lowering therapy, particularly in patients with type 2 diabetes at a high risk for cardiovascular events, may be associated with adverse cardiovascular outcomes [5]. Based on these results, the management of diabetes needs to consider not only glycemic control but also various complications, and it is extremely important to avoid hypoglycemia and weight gain.

Sodium-glucose cotransporter 2 (SGLT2) inhibitors enhance urinary glucose excretion, which consequently reduces hyperglycemia [6]. They exert favorable effects on various biomarkers, including blood glucose, body weight [7], blood pressure [8], albuminuria $[9,10]$, and fatty liver $[11,12]$. In addition to these effects, several large placebo-controlled cardiovascular and renal outcome trials in patients with type 2 diabetes have revealed that SGLT2 inhibitors may reduce the risk of heart failure, diabetic kidney disease, and cardiovascular death [13-17]. Based on these findings, in recent years, American and European diabetes and cardiology society guidelines and consensus reports recommend the use of SGLT2 inhibitors for patients with type 2 diabetes who have atherosclerotic cardiovascular disease (ASCVD) or those at risk [18, 19]. Although SGLT2 inhibitors have become an early recommended drug for patients with type 2 diabetes 
at a high risk of cardiovascular events, its effectiveness as a first-line medication for Japanese patients with newly diagnosed type 2 diabetes with no history of cardiovascular events is unclear.

Accordingly, the aim of the present study was to compare the efficacy and tolerability of dapagliflozin, an SGLT2 inhibitor, with those of sitagliptin and metformin, on plasma glucose and insulin levels and related biomarkers and hormones during a meal tolerance test (MTT) in drug-naïve Japanese patients with type 2 diabetes.

\section{METHODS}

\section{Participants}

We enrolled participants from the Ogawa Red Cross Hospital in Japan between July 2017 and May 2020. The participants included in the study were adult patients aged 20 years or older with type 2 diabetes who had never received glucose-lowering agents and had a body mass index (BMI) of $18-40 \mathrm{~kg} / \mathrm{m}^{2}$ besides a glycated hemoglobin (HbA1c) level between 6.5 and $11.0 \%$. The exclusion criteria included an estimated glomerular filtration rate (eGFR) of $<45 \mathrm{ml} / \mathrm{min} / 1.73 \mathrm{~m}^{2}$, serum creatinine level $>1.5 \mathrm{mg} / \mathrm{dl}$, history of serious diabetic complications, findings suggestive of insulin dependency, heart failure, and history of myocardial or cerebral infarction. This study complied with the Declaration of Helsinki and Good Clinical Practice guidelines and was approved by the Institutional Review Board of Ogawa Red Cross Hospital (no. OGAWARIN029). All participants provided written informed consent prior to enrollment.

\section{Study Design}

This was a randomized, 12-week, open-label, single-center, active-controlled trial. This trial was registered with the University Hospital Medical Information Network Clinical Trials Registry (UMIN000024427). Eligible participants were randomly assigned (1:1:1) using a computer-generated block randomization sequence to receive dapagliflozin, sitagliptin, or metformin for 12 weeks of study treatment. During the 4-week run-in period, all participants underwent diet and exercise therapy. At the start of the treatment period, all participants were administered Japanese standard dosages of dapagliflozin $(5 \mathrm{mg})$ or sitagliptin $(50 \mathrm{mg}$ ) orally once daily before breakfast and metformin $(500 \mathrm{mg})$ orally twice daily (1000 mg per day) before breakfast and dinner; no dosage adjustments were made during the study. However, if the participants in the metformin group who received $1000 \mathrm{mg}$ per day as an initial dose experienced side effects, such as diarrhea, then the dose was decreased to $500 \mathrm{mg}$ per day within 7 days. The dosages of these glucoselowering agents may be approximately half of those administered in Western countries. However, we intentionally adopted the standard dosages of these agents because our aim was to determine a suitable first-line medication.

\section{Meal Tolerance Tests}

At the start of the 12-week treatment period and at the end, the participants underwent an MTT. After basal blood sampling, the participants consumed a Japanese food test meal comprising white rice, a main dish, side dish, salad, and miso soup. The total energy content of the test meal was approximately $480 \mathrm{kcal}$, with $73.2 \mathrm{~g}$ of carbohydrates, $20.5 \mathrm{~g}$ of protein, and $10.7 \mathrm{~g}$ of fat $(60 \%$ carbohydrates, $18 \%$ protein, and $22 \%$ fat). Blood samples were obtained 60 and $120 \mathrm{~min}$ after the meal began. At the end of the treatment period, we confirmed that the participants took the study drugs before having the test meal. The test meals were provided by the Department of Nutrition, Ogawa Red Cross Hospital. A similar menu was offered to all participants.

\section{Study Outcomes}

All patients underwent an assessment of height, body weight, and blood pressure, besides providing blood samples for routine biochemical 
tests, including lipid level measurement and liver and renal function analyses.

The primary outcomes were considered as changes from the patient's baseline fasting and postprandial plasma glucose at week 12 . The key secondary outcomes were considered as changes in HbA1c, body weight, fasting and postprandial plasma insulin, plasma glucose area under the curve 0-120 min $\left(\mathrm{AUC}_{0-120 \mathrm{~min}}\right)$, insulin $\mathrm{AUC}_{0-120 \mathrm{~min}}$, fasting plasma proinsulin/ insulin ratio, and serum adiponectin at week 12 from the patient's baseline. Additional outcomes included changes in homeostasis model assessment of $\beta$-cell function (HOMA- $\beta$ ), homeostasis model assessment of insulin resistance (HOMA-IR), serum creatinine, eGFR, hematocrit, serum lipids, hepatobiliary enzymes, and blood pressure at week 12 from baseline.

\section{Statistical Analysis}

Data are presented as mean \pm standard deviation (SD). The Kruskal-Wallis test and Bonferroni correction were used to assess differences among the three study groups at baseline and at week 12 and in their respective changes from baseline. Moreover, changes in continuous measures between baseline and after the 12-week treatment period were tested using the Wilcoxon signed-rank test in each study group. The relationship between changes in glycemic and metabolic parameters was assessed using Spearman's rank correlation coefficients. Statistical significance was set at $P<0.05$. All analyses were performed using SPSS Statistics, version 27.0 (IBM Corp., Armonk, NY, USA).

\section{RESULTS}

\section{Patients}

Patient disposition is summarized in Table 1. Of the 39 patients who provided informed consent, 32 patients were randomly assigned to receive dapagliflozin $5 \mathrm{mg}(n=11)$, sitagliptin $50 \mathrm{mg}(n=11)$, or metformin $1000 \mathrm{mg}(n=10)$. Thirty-one patients completed the study (dapagliflozin group, $n=11$; sitagliptin group, $n=10$; metformin group, $n=10$ ). One patient in the sitagliptin group decided to withdraw before the treatment period, and the reason for discontinuation was recorded as patient

Table 1 Baseline characteristics of the study participants

\begin{tabular}{lllll}
\hline & $\begin{array}{l}\text { Dapagliflozin group } \\
(\boldsymbol{n}=\mathbf{1 1})\end{array}$ & $\begin{array}{l}\text { Sitagliptin group } \\
(\boldsymbol{n}=\mathbf{1 0})\end{array}$ & $\begin{array}{l}\text { Metformin group } \\
(\boldsymbol{n}=\mathbf{1 0})\end{array}$ & $\boldsymbol{P}$ value \\
\hline Age (years) & $55.9 \pm 7.5$ & $53.8 \pm 13.2$ & $57.5 \pm 9.6$ & 0.819 \\
Sex, male/female, $(n)$ & $8 / 3$ & $6 / 4$ & $9 / 1$ & 0.306 \\
Body weight $(\mathrm{kg})$ & $77.5 \pm 18.1$ & $77.3 \pm 24.8$ & $74.8 \pm 8.7$ & 0.965 \\
Body mass index $\left(\mathrm{kg} / \mathrm{m}^{2}\right)$ & $27.7 \pm 4.9$ & $27.1 \pm 6.6$ & $26.7 \pm 3.4$ & 0.891 \\
HbA1c (\%) & $7.86 \pm 0.93$ & $8.01 \pm 1.53$ & $7.93 \pm 0.92$ & 0.899 \\
Fasting plasma glucose $(\mathrm{mg} / \mathrm{dl})$ & $146.4 \pm 31.2$ & $161.7 \pm 56.5$ & $146.5 \pm 41.2$ & 0.508 \\
Fasting C-peptide $(\mathrm{ng} / \mathrm{ml})$ & $2.55 \pm 0.76$ & $2.34 \pm 0.93$ & $2.36 \pm 0.65$ & 0.814 \\
Nondiabetic complications, $n(\%)$ & & & & 0.544 \\
Hypertension & $2(18.2 \%)$ & $3(30.0 \%)$ & $4(40.0 \%)$ & - \\
Hyperlipidemia & $0(0.0 \%)$ & $0(0.0 \%)$ & $0(0.0 \%)$ & \\
\hline
\end{tabular}

Data are $n(\%)$, means \pm standard deviation (SD)

HbAlc glycated hemoglobin 
request. The baseline characteristics were similar among the three study groups. In one patient in the metformin group, the dose was temporarily reduced to $500 \mathrm{mg}$ per day within 7 days to address adverse events such as diarrhea that occurred during the treatment period. None of the patients discontinued the study drugs during the treatment period.

\section{Effects of Plasma Glucose and Insulin in the Meal Tolerance Test}

The changes in the time courses of plasma glucose and insulin after the MTT at baseline and at week 12 in the three study groups are shown in Fig. 1. Fasting and postprandial plasma glucose levels at 60 and $120 \mathrm{~min}$ after the start of the meal were significantly improved in the three study groups after the 12-week treatment plan, and there were no significant differences among the three study groups (Fig. 1A). In addition,

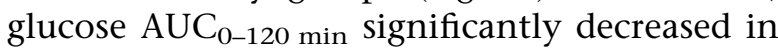
the dapagliflozin, sitagliptin, and metformin groups $(P=0.008, P=0.005$, and $P=0.007$, respectively) (Fig. $2 \mathrm{~A}$ and Table 2). Compared with baseline, the postprandial plasma insulin level at 120 min after the start of the meal at week 12 significantly decreased in the dapagliflozin and metformin groups $(P=0.004$ and $P=0.028$, respectively) (Fig. 1B). Furthermore, the insulin $\mathrm{AUC}_{0-120}$ min at week 12 was significantly decreased only in the dapagliflozin group $(P=0.049)$, and there were no significant differences among the three study groups $(P=0.501)$ (Fig. 2B and Table 2).

\section{Effects of Glycemic and Metabolic Parameters}

Table 2 presents the changes in glycemic and metabolic parameters from baseline to week 12 in the three study groups. The mean \pm SD change in HbA1c from baseline to week 12 were $-0.96 \pm 0.50 \% \quad(P=0.003),-1.24 \pm$ $0.74 \% \quad(P=0.007)$, and $-1.40 \pm 1.16 \% \quad(P=$ $0.005)$ in the dapagliflozin, sitagliptin, and metformin groups, respectively. Although there was no significant difference among the three study groups, the lowering effect of HbA1c tended to be greater in the metformin group
A

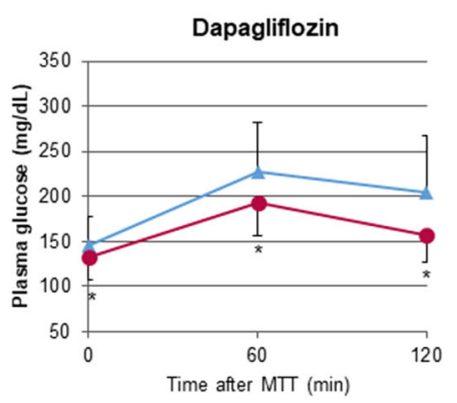

B

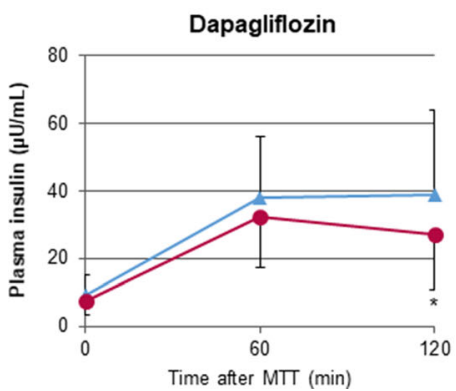

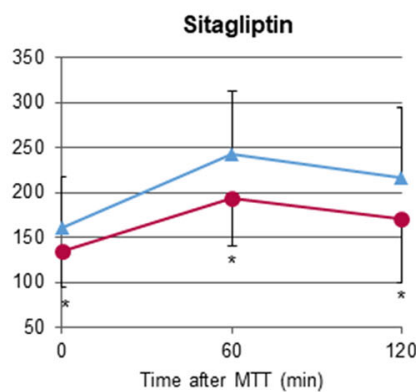
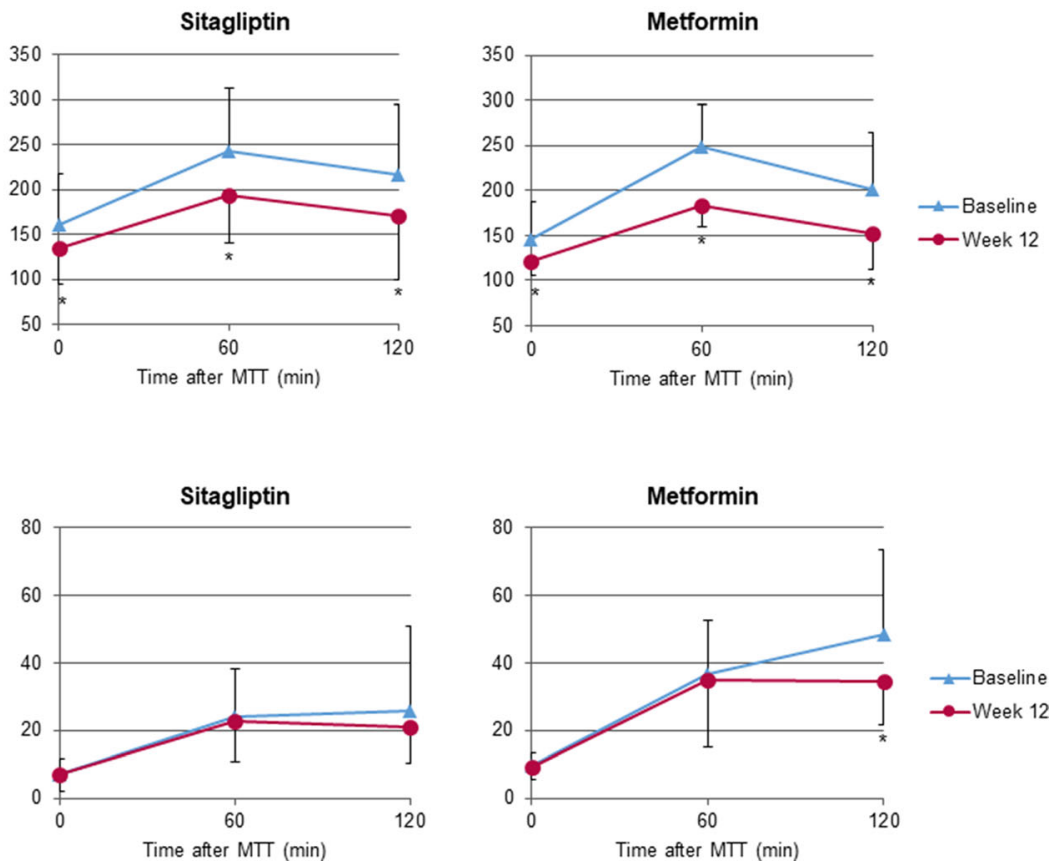

Fig. 1 Effects of dapagliflozin, sitagliptin, and metformin on the time course of plasma glucose (A) and plasma insulin (B) during the meal tolerance test. Data represent the mean \pm standard deviation 
A

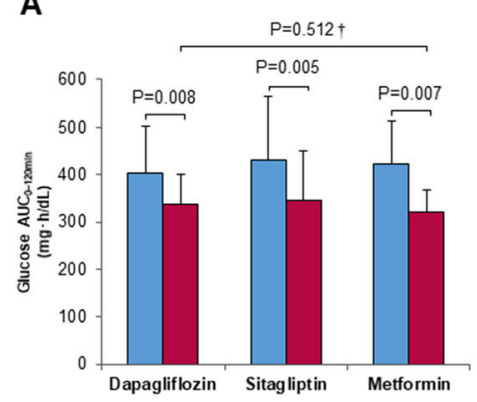

B

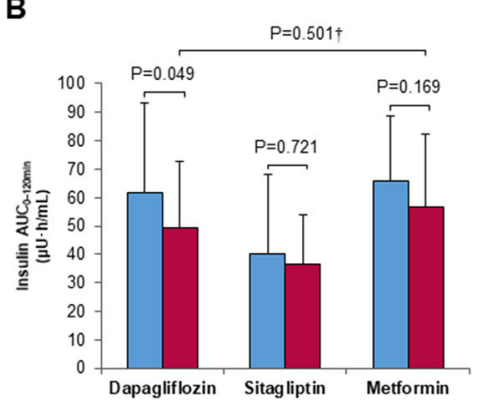

C

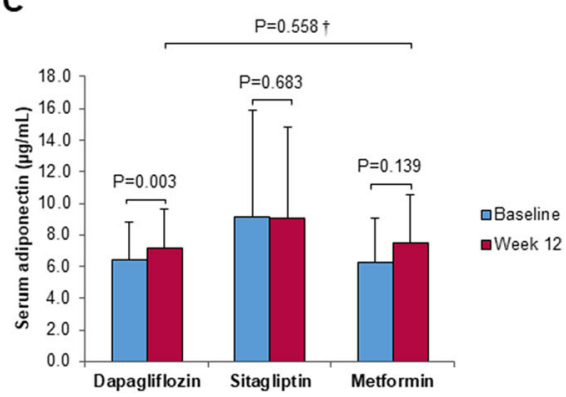

Fig. 2 Changes from baseline in plasma glucose area under the curve $0-120 \mathrm{~min}\left(\mathrm{AUC}_{0-120 \mathrm{~min}}\right)(\mathrm{A})$ and insulin $\mathrm{AUC}_{0-120 \text { min }}(\mathbf{B})$ during meal tolerance test and serum

than in the dapagliflozin group. In contrast, the changes in body weight from baseline to week 12 were $-2.1 \pm 2.9 \mathrm{~kg} \quad(P=0.047),-1.2 \pm$ $2.6 \mathrm{~kg} \quad(P=0.16), \quad$ and $-1.3 \pm 2.0 \mathrm{~kg} \quad(P=$ $0.074)$, respectively. Body weight was significantly reduced only in the dapagliflozin group. Similarly, the change in serum adiponectin level from baseline to week 12 increased significantly only in the dapagliflozin group $(P=0.003)$ (Fig. 2C and Table 2). However, there were no significant differences in the changes in these metabolic parameters among the three study groups.

The mean changes in the fasting proinsulin/ insulin ratio and HOMA- $\beta$ values from baseline to week 12 , as markers of $\beta$-cell function, are presented in Table 2. Although there were no significant differences from baseline to week 12, the changes in the proinsulin/insulin ratio tended to improve in the three study groups.

Notably, the changes in the hepatobiliary enzymes, including aspartate aminotransferase (AST), alanine aminotransferase (ALT), and $\gamma$-glutamyltransferase $(\gamma$-GT $), \quad$ significantly decreased from baseline to week 12 in the dapagliflozin and metformin groups. In contrast, the change in the hematocrit level from baseline to week 12 was significantly higher in the dapagliflozin group than in the sitagliptin and metformin groups $(P=0.030)$. There were no significant differences among the three study groups in terms of changes in renal function, serum lipid levels, and blood pressure (Table 2). adiponectin level (C) at week 12. Data represent the mean \pm standard deviation. ${ }^{\dagger}$ Comparison of the changes after 12 weeks from baseline among the three study groups
The extent of changes resulting from these treatments and their respective correlations with glycemic and metabolic parameters are presented in Table 3. We identified significant correlations between the changes in BMI and plasma glucose levels (i.e., fasting plasma glucose, postprandial plasma glucose level at 120 min after the start of the meal, and plasma glucose $\mathrm{AUC}_{0-120 \mathrm{~min}}$ ) only in the dapagliflozin group.

\section{Subgroup Analysis Based on BMI}

Figure 3 shows the subgroup analysis results for patients categorized according to their BMI at baseline into two groups: low (BMI approximately $<25 \mathrm{~kg} / \mathrm{m}^{2}$ ) and high (BMI approximately $>25 \mathrm{~kg} / \mathrm{m}^{2}$ ) in each study group. The changes in HbA1c from baseline to week 12 improved in all three study groups regardless of BMI (Fig. 3A). In the dapagliflozin group, the changes in body weight from baseline to week 12 were $-1.4 \pm 1.1 \mathrm{~kg}(-2.0 \%)$ in the low BMI group and $-2.9 \pm 4.2 \mathrm{~kg}(-3.3 \%)$ in the high BMI group; the weight loss effect was greater in patients with higher obesity. Notably, significant weight loss was observed only in low BMI individuals in the dapagliflozin group $(P=0.043)$. The sitagliptin and metformin groups showed no significant weight changes regardless of BMI (Fig. 3B). In the dapagliflozin group, a marked improvement in plasma glucose $\mathrm{AUC}_{0-120 \text { min }}$ was observed in the high BMI group. In contrast, in the metformin group, a 
Table 2 Effects of dapagliflozin and sitagliptin vs. metformin on glycemic and metabolic parameters at 12 weeks

\begin{tabular}{llll}
\hline $\begin{array}{l}\text { Dapagliflozin group } \\
(n=11)\end{array}$ & $\begin{array}{l}\text { Sitagliptin group } \\
(n=10)\end{array}$ & $\begin{array}{l}\text { Metformin group } \\
(n=10)\end{array}$ & $P$ value
\end{tabular}

HbAlc (\%)

Baseline

Week 12

Change from baseline ${ }^{\dagger}$

Body weight $(\mathrm{kg})$

Baseline

Week 12

Change from baseline

Fasting plasma glucose (mg/dl)

Baseline

$146.4 \pm 31.2$

Week 12

$132.5 \pm 63.0$

Change from baseline

$-13.9 \pm 16.6^{*}$

Glucose $\mathrm{AUC}_{0-120 \mathrm{~min}}(\mathrm{mg} \cdot \mathrm{h} / \mathrm{dl})$

Baseline

$402.8 \pm 100.9$

Week 12

Change from baseline

$337.8 \pm 61.7$

$-65.0 \pm 65.6^{*}$

Fasting plasma insulin $(\mu \mathrm{U} / \mathrm{ml})$

Baseline

$9.13 \pm 6.04$

Week 12

$7.45 \pm 4.14$

Change from baseline

$-1.68 \pm 3.22$

Insulin $\mathrm{AUC}_{0-120 \min }(\mu \mathrm{U} \cdot \mathrm{h} / \mathrm{ml})$

Baseline

Week 12

$61.9 \pm 31.5$

$49.6 \pm 23.1$

Change from baseline

Fasting proinsulin/insulin ratio
Baseline
$4.17 \pm 4.30$
Week 12
$3.14 \pm 1.63$
Change from baseline
$-1.03 \pm 2.96$

Serum adiponectin $(\mu \mathrm{g} / \mathrm{ml})$

Baseline

$6.40 \pm 2.41$

$7.16 \pm 2.45$

$0.76 \pm 0.46^{*}$

Week 12

Change from baseline
$-12.3 \pm 18.1^{*}$

$$
\begin{array}{r}
8.01 \pm 1.53 \\
6.77 \pm 1.40 \\
-1.24 \pm 0.74^{*}
\end{array}
$$

$$
7.93 \pm 0.92
$$

$6.53 \pm 0.54$

$-1.40 \pm 1.16^{*}$

0.562

$$
\begin{aligned}
77.3 & \pm 24.8 \\
76.1 & \pm 23.8 \\
-1.2 & \pm 2.6
\end{aligned}
$$

\begin{tabular}{|c|c|c|}
\hline $161.7 \pm 56.5$ & $146.5 \pm 41.2$ & 0.508 \\
\hline $134.8 \pm 40.8$ & $120.6 \pm 14.4$ & 0.604 \\
\hline $26.9 \pm 21.5^{*}$ & $-25.9 \pm 41.0^{*}$ & 0.435 \\
\hline
\end{tabular}$$
74.8 \pm 8.7
$$$$
0.965
$$$$
73.5 \pm 8.6
$$$$
0.987
$$$$
-1.3 \pm 2.0
$$

$$
\begin{aligned}
& 431.9 \pm 132.6 \\
& 345.4 \pm 103.7
\end{aligned}
$$$$
423.3 \pm 89.9
$$$$
320.4 \pm 47.2
$$$$
-86.5 \pm 48.8^{*}
$$$$
-103.0 \pm 93.5^{*}
$$

$$
\begin{aligned}
& 6.98 \pm 4.75 \\
& 7.14 \pm 5.34 \\
& 0.16 \pm 3.40
\end{aligned}
$$$$
9.54 \pm 3.72
$$

$9.04 \pm 3.34$

0.354

$-0.50 \pm 2.93$

0.589

$40.3 \pm 27.7$

$65.6 \pm 23.3$

0.067

$36.6 \pm 17.6$

$56.5 \pm 25.8$

0.234

$-3.7 \pm 17.0$

$-9.1 \pm 19.5$

0.501
$2.24 \pm 0.83$

0.137

$3.16 \pm 1.90$

$-0.69 \pm 1.06$

$1.85 \pm 0.95$

0.077

$-0.38 \pm 0.77$

0.599

0.76


Table 2 continued

\section{Dapagliflozin \\ $\operatorname{group}(n=11)$}

HOMA- $\beta$ (\%)

Baseline

Week 12

Change from baseline

HOMA-IR

Baseline

Week 12

Change from baseline

Serum creatinine $(\mathrm{mg} / \mathrm{dl})$

Baseline
Week 12
Change from baseline
eGFR $\left(\mathrm{ml} / \mathrm{min} / 1.73 \mathrm{~m}^{2}\right)$

Baseline

Week 12

Change from baseline

Hematocrit (\%)

Baseline

Week 12

Change from baseline

Total cholesterol $(\mathrm{mg} / \mathrm{dl})$

Baseline

Week 12

Change from baseline

LDL cholesterol $(\mathrm{mg} / \mathrm{dl})$

Baseline

Week 12

Change from baseline

HDL cholesterol (mg/dl)

Baseline

Week 12

Change from baseline
$39.9 \pm 18.8$
$40.1 \pm 16.3$
$0.20 \pm 17.3$

$3.57 \pm 3.06$

$2.55 \pm 1.82$

$-1.02 \pm 1.47^{*}$

$0.77 \pm 0.16$

$0.82 \pm 0.18$

$0.05 \pm 0.09$

$77.6 \pm 11.5$

$73.3 \pm 13.0$

$-4.4 \pm 8.1$

$$
\begin{aligned}
45.6 & \pm 3.4 \\
47.3 & \pm 3.5 \\
1.7 & \pm 2.0^{*}
\end{aligned}
$$

$212.4 \pm 28.2$

$207.5 \pm 22.3$

$-4.8 \pm 14.9$

$124.0 \pm 28.3$

$120.9 \pm 18.7$

$-3.1 \pm 17.2$

$55.6 \pm 14.3$

$56.5 \pm 12.6$

$0.8 \pm 6.0$
Sitagliptin
$\operatorname{group}(n=10)$

Metformin

$\operatorname{group}(n=10)$

$P$ value

$28.1 \pm 19.3$

$45.6 \pm 19.0$

0.090

$42.9 \pm 36.0$

$60.2 \pm 27.4$

0.158

$14.8 \pm 21.3^{*}$

$14.6 \pm 23.8$

0.273

$2.90 \pm 2.13$

$3.46 \pm 1.53$

0.631

$2.46 \pm 2.17$

$2.71 \pm 1.17$

0.585

$-0.44 \pm 1.32$

$-0.75 \pm 1.46$

0.626

$0.67 \pm 0.16$

$0.88 \pm 0.21$

0.083

$0.73 \pm 0.13$

$0.87 \pm 0.22$

0.243

$0.06 \pm 0.06^{*}$

$-0.01 \pm 0.06$

0.066

$90.5 \pm 19.3$

$72.3 \pm 19.9$

0.114

$80.2 \pm 12.3$

$73.9 \pm 20.9$

0.447

$1.6 \pm 7.1$

0.053

$-10.3 \pm 10.7^{*}$

$44.0 \pm 3.4$

$44.0 \pm 5.0$

0.500

$43.8 \pm 2.8$

$43.1 \pm 5.2$

0.031

$-0.1 \pm 1.8$

$-0.9 \pm 3.2$

0.030

$198.4 \pm 41.9$

$201.4 \pm 38.3$

0.451

$197.4 \pm 51.2$

$195.3 \pm 42.5$

0.541

$-1.0 \pm 20.9$

$-6.1 \pm 16.7$

0.810

$110.3 \pm 30.2$

$137.8 \pm 23.5$

0.190

$120.2 \pm 41.4$

$128.7 \pm 28.5$

0.834

$9.9 \pm 24.0$

$-9.1 \pm 13.3$

0.098

$60.1 \pm 22.9$

$42.0 \pm 9.9$

0.060

$60.7 \pm 23.4$

$49.3 \pm 12.8$

0.340

$0.6 \pm 7.7$

$7.3 \pm 12.6^{*}$

0.198 
Table 2 continued

$\begin{array}{lll}\text { Dapagliflozin } & \text { Sitagliptin } & \text { Metformin } \\ \operatorname{group}(n=11) & \operatorname{group}(n=10) & \operatorname{group}(n=10)\end{array}$

Triglycerides (mg/dl)

Baseline

Week 12

Change from baseline

$\operatorname{AST}(\mathrm{U} / \mathrm{l})$

Baseline

Week 12

Change from baseline

$\operatorname{ALT}(\mathrm{U} / \mathrm{l})$

Baseline

Week 12

Change from baseline

$\gamma-\mathrm{GT}(\mathrm{U} / \mathrm{l})$

Baseline

Week 12

Change from baseline

$$
\begin{gathered}
168.9 \pm 138.2 \\
148.1 \pm 69.1 \\
-20.8 \pm 88.4
\end{gathered}
$$$$
28.8 \pm 12.8
$$$$
25.5 \pm 12.2
$$$$
-3.4 \pm 3.8^{*}
$$

$36.2 \pm 18.1$

$25.7 \pm 13.0$

$-10.5 \pm 12.8^{*}$

$$
73.5 \pm 62.5
$$$$
60.9 \pm 56.1
$$$$
-12.6 \pm 15.2^{*}
$$

Systolic blood pressure $(\mathrm{mmHg})$

$$
\text { Baseline }
$$

$$
137.1 \pm 17.6
$$

Week 12

$$
132.4 \pm 12.5
$$

Change from baseline

$$
-4.7 \pm 11.1
$$

Diastolic blood pressure $(\mathrm{mmHg})$

\begin{abstract}
Baseline
\end{abstract}
$86.1 \pm 13.6$

$88.3 \pm 9.2$

$2.2 \pm 12.6$

$$
\begin{gathered}
151.9 \pm 136.0 \\
103.0 \pm 74.2 \\
-48.9 \pm 74.2
\end{gathered}
$$

$25.5 \pm 6.8$

$24.5 \pm 4.2$

$-1.0 \pm 6.3$

$26.1 \pm 8.7$

$23.5 \pm 5.6$

$-2.6 \pm 6.4$

$46.6 \pm 65.3$

$33.1 \pm 35.2$

$-13.5 \pm 30.5$

$$
134.4 \pm 14.9
$$

$127.5 \pm 13.3$

$-6.9 \pm 14.1$

$$
85.5 \pm 9.2
$$$$
84.7 \pm 12.9
$$

$-0.8 \pm 10.0$

$$
28.0 \pm 17.1
$$

$130.9 \pm 52.7$

$115.1 \pm 63.6$

$-15.8 \pm 41.7$$$
21.6 \pm 9.2
$$$$
-6.4 \pm 10.8^{*}
$$

0.698

0.532

$-11.2 \pm 19.4^{*}$

$-47.2 \pm 94.0^{*}$

Data are mean \pm standard deviation $(\mathrm{SD}) .{ }^{*} P \leq 0.05$ compared with the baseline. ${ }^{\dagger}$ Extent of changes 12 weeks after baseline $\pm \mathrm{SD} .{ }^{\ddagger}$ Comparison of the changes after 12 weeks from baseline among the three study groups

$H b A 1 c$ glycated hemoglobin, $A U C$ area under the curve, $H O M A-\beta$ homeostasis model assessment of $\beta$-cell function, HOMA-IR homeostasis model assessment of insulin resistance, eGFR estimated glomerular filtration rate, $A S T$ aspartate aminotransferase, $A L T$ alanine aminotransferase, $\gamma$-GT $\gamma$-glutamyltransferase

marked improvement in plasma glucose AUC $_{0-120 \text { min }}$ was observed in the lower BMI group (Fig. 3C). A significant decrease in insulin
AUC $_{0-120}$ min was observed only in the high BMI subgroup of those treated with dapagliflozin (Fig. 3D). 
Table 3 Correlations of changes in glycemic and metabolic parameters

\begin{tabular}{|c|c|c|c|c|c|c|c|c|}
\hline & \multicolumn{2}{|c|}{$\Delta B M I\left(\mathrm{~kg} / \mathrm{m}^{2}\right)$} & \multicolumn{2}{|c|}{$\Delta$ Adiponectin $(\mu \mathrm{g} / \mathrm{ml})$} & \multicolumn{2}{|c|}{ 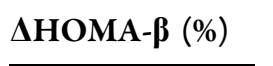 } & \multicolumn{2}{|c|}{ DHOMA-IR } \\
\hline & $r$ & $p$ & $r$ & $p$ & $r$ & $p$ & $r$ & $p$ \\
\hline \multicolumn{9}{|l|}{ Dapagliflozin group $(n=11)$} \\
\hline$\Delta \mathrm{FPG}(\mathrm{mg} / \mathrm{dl})$ & 0.655 & 0.029 & -0.429 & 0.188 & -0.382 & 0.247 & 0.382 & 0.247 \\
\hline$\Delta 2-\mathrm{h}$ PPG $(\mathrm{mg} / \mathrm{dl})$ & 0.645 & 0.032 & -0.313 & 0.348 & -0.064 & 0.853 & 0.582 & 0.060 \\
\hline$\Delta$ Glucose AUC $(\mathrm{mg} \cdot \mathrm{h} / \mathrm{dl})$ & 0.718 & 0.013 & -0.387 & 0.239 & -0.500 & 0.117 & 0.282 & 0.401 \\
\hline \multicolumn{9}{|l|}{ Sitagliptin group $(n=10)$} \\
\hline$\Delta \mathrm{FPG}(\mathrm{mg} / \mathrm{dl})$ & 0.109 & 0.763 & 0.689 & 0.028 & -0.426 & 0.220 & 0.049 & 0.894 \\
\hline$\Delta 2-\mathrm{h}$ PPG $(\mathrm{mg} / \mathrm{dl})$ & 0.042 & 0.907 & 0.353 & 0.318 & 0.042 & 0.907 & 0.273 & 0.446 \\
\hline$\Delta$ Glucose AUC $(\mathrm{mg} \cdot \mathrm{h} / \mathrm{dl})$ & 0.212 & 0.556 & 0.638 & 0.047 & -0.539 & 0.108 & -0.200 & 0.580 \\
\hline \multicolumn{9}{|l|}{ Metformin group $(n=10)$} \\
\hline$\Delta \mathrm{FPG}(\mathrm{mg} / \mathrm{dl})$ & 0.626 & 0.053 & -0.176 & 0.626 & -0.632 & 0.050 & 0.675 & 0.032 \\
\hline$\Delta 2-\mathrm{h}$ PPG $(\mathrm{mg} / \mathrm{dl})$ & 0.236 & 0.511 & -0.576 & 0.082 & -0.406 & 0.244 & 0.394 & 0.260 \\
\hline$\Delta$ Glucose AUC $(\mathrm{mg} \cdot \mathrm{h} / \mathrm{dl})$ & 0.491 & 0.150 & -0.600 & 0.067 & -0.600 & 0.067 & 0.370 & 0.293 \\
\hline
\end{tabular}

The relationship of changes in glycemic and metabolic parameters were assessed using Spearman's rank correlation coefficients. $\Delta$, the amount of change 12 weeks after baseline; $F P G$ fasting plasma glucose, $2-h P P G$ postprandial plasma glucose level at $120 \mathrm{~min}$ after the start of the meal, Glucose $A U C$ postprandial glucose area under the curve $0-120$ min, $B M I$ body mass index, HOMA- $\beta$ homeostasis model assessment of $\beta$-cell function, HOMA-IR homeostasis model assessment of insulin resistance

\section{Safety}

During the 12-week treatment period, adverse events were documented in two patients. One woman in the dapagliflozin group developed urinary tract infection, while another in the metformin group experienced diarrhea. However, these symptoms resolved upon appropriate administration of antibiotics and temporary reduction of study drug dose, respectively, allowing the patients to continue the study. No serious adverse effects that necessitated treatment interruption or participant termination, such as severe hypoglycemia, dehydration, ketoacidosis, heart failure, renal failure, or severe infections, were recorded.

\section{DISCUSSION}

Currently, the first-line medications for type 2 diabetes have not been clarified in the guidelines or algorithms in Japan. Thus, the present study aimed to determine whether dapagliflozin is a suitable first-line medication for newly diagnosed patients with type 2 diabetes. In the present study, we performed an MTT to compare the response of plasma glucose levels and related variable parameters after dapagliflozin treatment with that after sitagliptin and metformin treatment. Additionally, we selected food for the test meal that mimicked a typical Japanese breakfast, because we hoped to verify the effectiveness and safety of each study drug in accordance with actual clinical practice.

The present study revealed that dapagliflozin exerts a glucose-lowering effect comparable to that of the alternative first-line medications, metformin and sitagliptin. Besides its hypoglycemic action, dapagliflozin significantly lowered body weight and increased serum adiponectin levels in participants. It has also been suggested that dapagliflozin may protect pancreatic $\beta$ cells by diminishing plasma insulin 
A

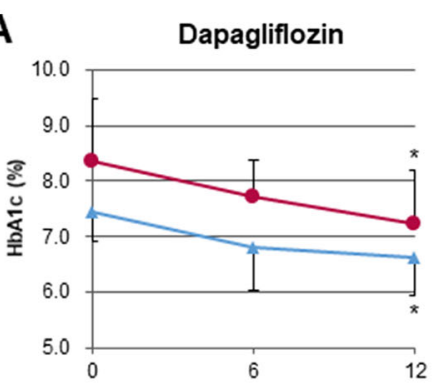

B

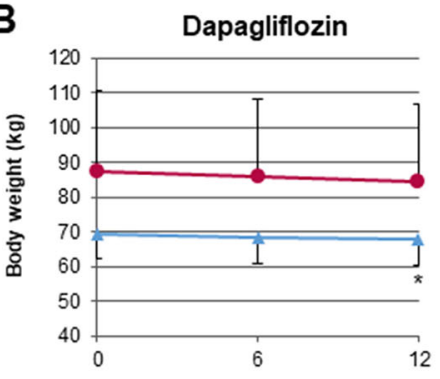

C

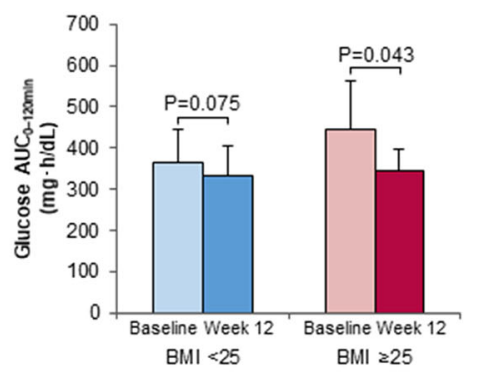

D

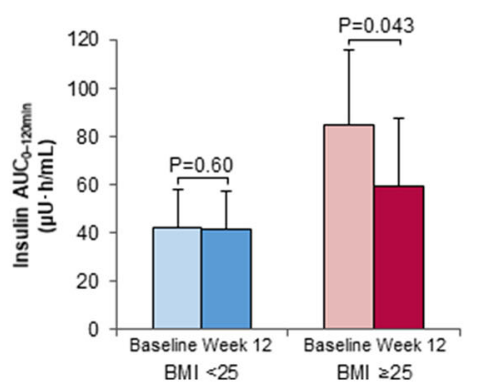

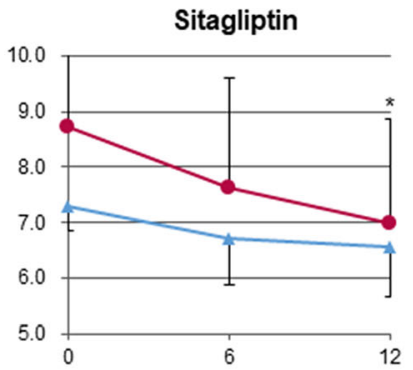
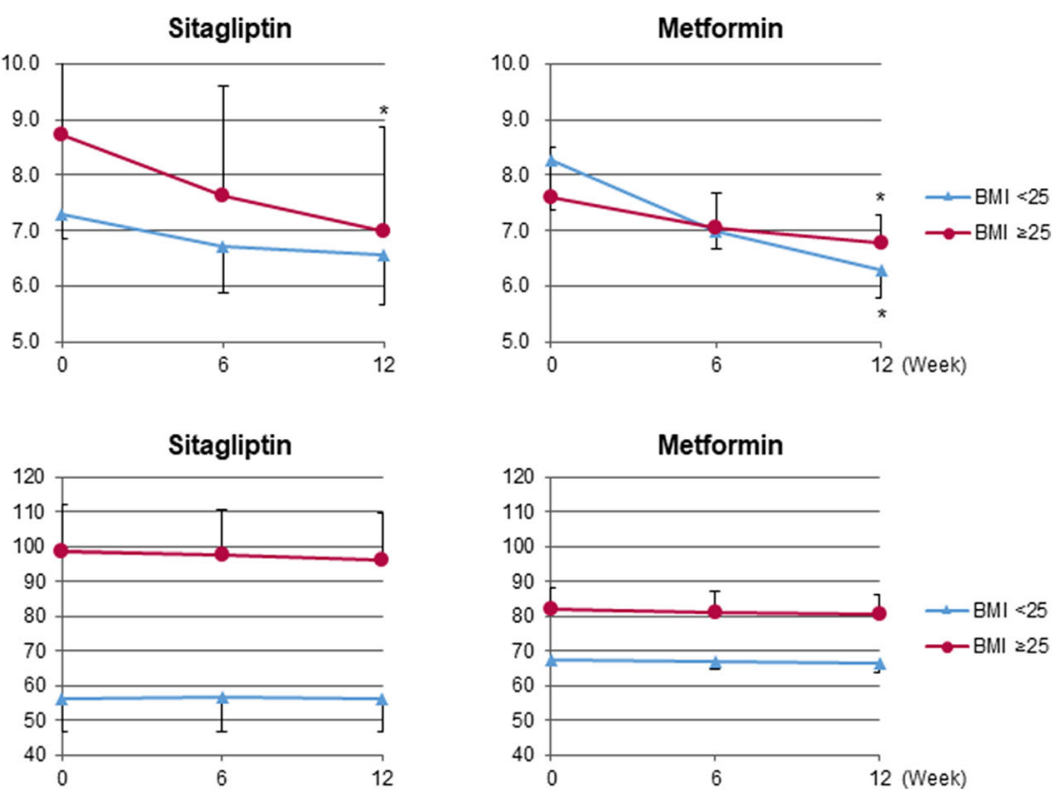

Sitagliptin

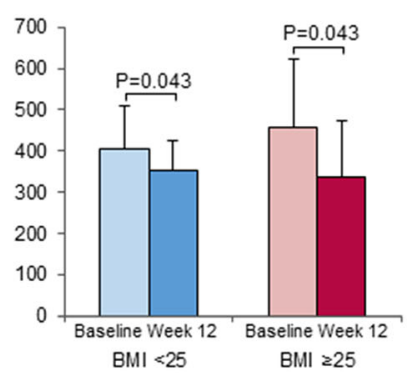

Sitagliptin

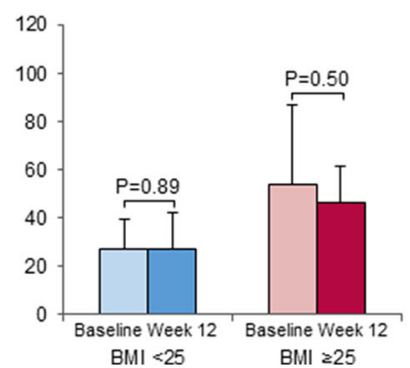

Metformin

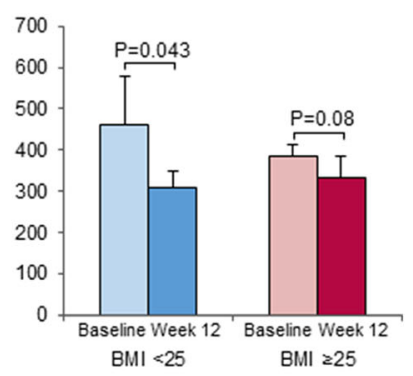

Metformin

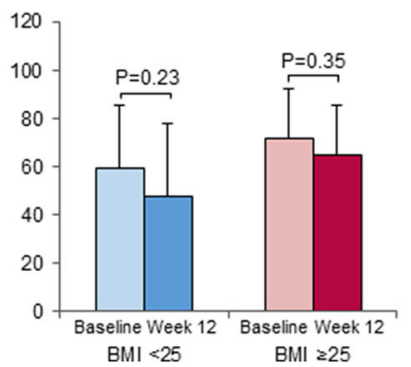

Fig. 3 Changes from baseline in $\mathrm{HbAlc}(\mathbf{A})$, body weight (B), plasma glucose area under the curve 0-120 min $\left(\mathrm{AUC}_{0-120 \mathrm{~min}}\right)(\mathbf{C})$, and insulin $\mathrm{AUC}_{0-120 \text { min }}(\mathbf{D})$ during meal tolerance test at week 12 considering subgroup analysis based on body mass index. Data represent the mean \pm standard deviation 
levels, which may be a pivotal feature to control type 2 diabetes patients for a long time.

Based on evidence from the UKPDS [2], metformin has long been the first-line medication for the management of type 2 diabetes in Europe and the US. The consensus report by the American Diabetes Association and the European Association for the Study of Diabetes (2018) stated that metformin is the preferred glucose-lowering medication for newly diagnosed patients with type 2 diabetes [18]. In contrast, the consensus report suggests considering the use of SGLT2 inhibitors in patients with type 2 diabetes and ASCVD, heart failure, and chronic kidney disease (CKD), based on new evidence that SGLT2 inhibitors improve cardiovascular outcomes and renal disease progression [13-15].

In contrast, in Japan, dipeptidyl peptidase- 4 (DPP-4) inhibitors are currently the most commonly used first-line medications for the treatment of type 2 diabetes. Regarding the difference in the frequency of use of glucoselowering agents, the difference in the pathophysiology of type 2 diabetes was first mentioned by comparing Westerners and Asians. That is, while insulin resistance associated with obesity is the main factor in the pathophysiology of type 2 diabetes in Westerners, Asians, including the Japanese, are more vulnerable to insulin secretion from pancreatic $\beta$ cells [20]. Next, the frequency of cardiovascular events in diabetes patients is different between Western and Japanese patients. When comparing the evidence provided by the UKPDS [2], the Action to Control Cardiovascular Risk in Diabetes (ACCORD) trial [21], and the Action in Diabetes and Vascular Disease: Preterax and Diamicron Modified Release Controlled Evaluation (ADVANCE) trial [22] regarding Europe and the US, and the Japan Diabetes Complications Study (JDCS) [23] and J-DOIT3 [4] regarding Japan, there was no difference in the frequency of stroke in patients with diabetes; however, there were clearly fewer Japanese patients who experienced cardiovascular death and myocardial infarction. In addition, the facts that the average life expectancy of Japanese is $>84$ years and most diabetes patients are elderly are likely to lead to differences in the frequency of using glucose-lowering agents.

Considering these backgrounds, when choosing medications to act as first-line medications for patients with type 2 diabetes, we believe that it is important that the agent has a good glucose-lowering effect, does not cause hypoglycemia or weight gain, protects pancreatic $\beta$ cells, and is highly tolerable, especially in elderly diabetes patients. Moreover, it is vital to reduce cardiovascular events, especially heart failure. In our present study, the study participants had an average age of 55.7 years, and several patients were around 70 years old; dapagliflozin was well tolerated to a similar extent as the other two study drugs. Considering the beneficial effects of dapagliflozin on blood glucose and body weight, as well as the multifaceted effects of SGLT2 inhibitors on ASCVD, heart failure, CKD, and fatty liver, we believe that SGLT2 inhibitors may fulfill the requirements for a first-line medication in Japanese patients with type 2 diabetes.

Recently, several clinical studies have evaluated the efficacy of SGLT2 inhibitors, especially using MTT [24-26]. However, our present study is the first to directly compare these drugs in newly diagnosed patients with type 2 diabetes using MTT, with the aim of identifying the drugs that could be effectively and safely used in actual clinical practice. Moreover, the use of Japanese food in the test meal was considered very advantageous for evaluating the actual daily glycemic profile of Japanese people. In the present study, dapagliflozin did not significantly improve glycemic and metabolic parameters compared to metformin or DPP-4 inhibitors; therefore, we presume that these three hypoglycemic agents are all balanced firstline medications for patients with drug-naïve type 2 diabetes. Nonetheless, considering the increasing obesity level in diabetes patients in recent years, SGLT2 inhibitors that also target body weight might be more beneficial. In addition, considering the advanced age of many diabetes patients, high frequency of complications related to diabetes and heart failure, and poor prognosis of patients with diabetes and heart failure [27], we believe that SGLT2 inhibitors will be more desirable in the future than 
metformin, which is often difficult to administer to elderly diabetes patients with decreased renal function, and some DPP-4 inhibitors, which may increase the risk of heart failure $[28,29]$. In contrast, the present study showed that metformin has a greater HbA1c-lowering effect than dapagliflozin. This result does not indicate that dapagliflozin is a better drug than metformin for diabetes patients without cardiovascular risk, where glycemic control is important to prevent microvascular complications. This fact indicates the need for studies with a bigger sample size to demonstrate the definitive efficacy of SGLT2 inhibitors as firstline drugs.

The present study has some limitations. First, the sample size was small; it was performed at a single facility and with an open-label design. Second, because there have been few reports of clinical studies evaluating glycemic parameters of SGLT2 inhibitors using an MTT, no appropriate sample size calculations were performed. Third, the dosage of the study drugs, which is adopted as the standard dosage in Japan, is approximately half of that in Western countries. Finally, because the present study lasted only 12 weeks, the long-term efficacy of dapagliflozin as a first-line medication is unknown and needs to be evaluated over a longer period and in a larger group of participants.

\section{CONCLUSION}

Dapagliflozin exerted beneficial effects similar to sitagliptin and metformin on fasting and postprandial plasma glucose and glucose AUC levels during MTT as well as HbA1c level in drug-naïve Japanese patients with type 2 diabetes. Furthermore, dapagliflozin significantly reduced body weight and insulin AUC levels and improved serum adiponectin levels without inducing hypoglycemia. These results suggest that along with metformin and DPP-4 inhibitors, SGLT2 inhibitors could be a viable first-line treatment option for drug-naïve Japanese patients with type 2 diabetes because of their optimum glucose-lowering properties, ability to avoid hypoglycemia or weight gain, and tolerability over a wide range of ages.

\section{ACKNOWLEDGEMENTS}

We thank all participants, investigators, and trial staff.

Funding. No funding or sponsorship was received for this study or publication of this article. The Rapid Service Fee was funded by the authors.

Authorship. All named authors meet the International Committee of Medical Journal Editors (ICMJE) criteria for authorship for this article, take responsibility for the integrity of the work as a whole, and have given their approval for this version to be published.

Author Contributions. Daisuke Ito, Kazuyuki Inoue, Daigo Saito, and Takashi Sumita were trial investigators and helped with data collection. Keiko Hamaguchi, Kimie Kaneko, Ikuo Inoue, and Akira Shimada were the medical advisors for this study. Kouichi Inukai was the medical advisors for this study and contributed to the study design. Daisuke Ito performed the statistical analyses. All authors contributed to interpretation of data and review of the manuscript and approved this manuscript for submission.

Disclosures. Kouichi Inukai has received lecture fees from AstraZeneca K.K. and Ono Pharmaceutical Co., Ltd. Akira Shimada has received research support from Ono Pharmaceutical Co., Ltd., as the chief professor of the Department of Endocrinology and Diabetes, Saitama Medical University. Daisuke Ito, Kazuyuki Inoue, Daigo Saito, Keiko Hamaguchi, Kimie Kaneko, Takashi Sumita, and Ikuo Inoue has nothing to disclose.

Compliance with Ethics Guidelines. The study protocol was approved by the Institutional Review Board of Ogawa Red Cross Hospital in Japan (no. OGAWARIN029). All procedures followed were in accordance with the ethical standards of the responsible committee on human experimentation (institutional and national) and with the Helsinki Declaration of 1964, as revised in 2013. 
Informed consent was obtained from all individual participants included in the study.

Data Availability. The datasets generated during and/or analyzed during the current study are available from the corresponding author on reasonable request.

Open Access. This article is licensed under a Creative Commons Attribution-NonCommercial 4.0 International License, which permits any non-commercial use, sharing, adaptation, distribution and reproduction in any medium or format, as long as you give appropriate credit to the original author(s) and the source, provide a link to the Creative Commons licence, and indicate if changes were made. The images or other third party material in this article are included in the article's Creative Commons licence, unless indicated otherwise in a credit line to the material. If material is not included in the article's Creative Commons licence and your intended use is not permitted by statutory regulation or exceeds the permitted use, you will need to obtain permission directly from the copyright holder. To view a copy of this licence, visit http://creativecommons.org/licenses/by$\mathrm{nc} / 4.0 /$.

\section{REFERENCES}

1. Ministry of Health, Labour and Welfare, Japan. The National Health and Nutrition Survey in Japan 2016. https://www.mhlw.go.jp/stf/houdou/ 0000177189.html. Sep 21, 2017. Accessed June 27, 2021.

2. UK Prospective Diabetes Study (UKPDS) Group. Intensive blood-glucose control with sulphonylureas or insulin compared with conventional treatment and risk of complications in patients with type 2 diabetes (UKPDS 33). Lancet. 1998;352: 837-53.

3. Gaede P, Lund-Andersen H, Parving HH, Pedersen O. Effect of a multifactorial intervention on mortality in type 2 diabetes. N Engl J Med. 2008;358: 580-91.

4. Ueki K, Sasako T, Okazaki Y, et al. Effect of an intensified multifactorial intervention on cardiovascular outcomes and mortality in type 2 diabetes
(J-DOIT3): an open-label, randomised controlled trial. Lancet Diabetes Endocrinol. 2017;5:951-64.

5. Udell JA, Cavender MA, Bhatt DL, Chatterjee S, Farkouh ME, Scirica BM. Glucose-lowering drugs or strategies and cardiovascular outcomes in patients with or at risk for type 2 diabetes: a meta-analysis of randomised controlled trials. Lancet Diabetes Endocrinol. 2015;3:356-66.

6. Gallo LA, Wright EM, Vallon V. Probing SGLT2 as a therapeutic target for diabetes: basic physiology and consequences. Diabetes Vasc Dis Res. 2015;12: 78-89.

7. Vasilakou D, Karagiannis T, Athanasiadou E, et al. Sodium-glucose cotransporter 2 inhibitors for type 2 diabetes: a systematic review and meta-analysis. Ann Intern Med. 2013;159:262-74.

8. Baker WL, Smyth LR, Riche DM, Bourret EM, Chamberlin KW, White WB. Effects of sodiumglucose co-transporter 2 inhibitors on blood pressure: a systematic review and meta-analysis. J Am Soc Hypertens. 2014;8:262-75.

9. Cherney DZ, Perkins BA, Soleymanlou N, et al. Renal hemodynamic effect of sodium-glucose cotransporter 2 inhibition in patients with type 1 diabetes mellitus. Circulation. 2014;129:587-97.

10. Ito D, Ikuma-Suwa $\mathrm{E}$, Inoue $\mathrm{K}$, et al. Effects of Ipragliflozin on diabetic nephropathy and blood pressure in patients with type 2 diabetes: an openlabel study. J Clin Med Res. 2017;9:154-62.

11. Ito D, Shimizu S, Inoue $\mathrm{K}$, et al. Comparison of Ipragliflozin and pioglitazone effects on nonalcoholic fatty liver disease in patients with type 2 diabetes: a randomized, 24-week, open-label, active-controlled trial. Diabetes Care. 2017;40: 1364-72.

12. Chehrehgosha H, Sohrabi MR, Ismail-Beigi F, et al. Empagliflozin improves liver steatosis and fibrosis in patients with non-alcoholic fatty liver disease and type 2 diabetes: a randomized, double-blind, placebo-controlled clinical trial. Diabetes Ther. 2021;12:843-61.

13. Zinman B, Wanner C, Lachin JM, et al. Empagliflozin, cardiovascular outcomes, and mortality in type 2 diabetes. N Engl J Med. 2015;373:2117-28.

14. Wanner C, Inzucchi SE, Lachin JM, et al. EMPA-REG OUTCOME Investigators. Empagliflozin and progression of kidney disease in type 2 diabetes. $\mathrm{N}$ Engl J Med. 2016;375:323-34.

15. Neal B, Perkovic V, Mahaffey KW, et al. Canagliflozin and cardiovascular and renal events in type 2 diabetes. N Engl J Med. 2017;377:644-57. 
16. Wiviott SD, Raz I, Bonaca MP, et al. Dapagliflozin and cardiovascular outcomes in type 2 diabetes. N Engl J Med. 2019;380:347-57.

17. Perkovic V, Jardine MJ, Neal B, et al. Canagliflozin and renal outcomes in type 2 diabetes and nephropathy. N Engl J Med. 2019;380:2295-306.

18. Davies MJ, D'Alessio DA, Fradkin J, et al. Management of hyperglycaemia in type 2 diabetes, 2018. A consensus report by the American Diabetes Association (ADA) and the European Association for the Study of Diabetes (EASD). Diabetologia. 2018;2018(61):2461-98.

19. Cosentino F, Grant PJ, Aboyans V, et al. 2019 ESC Guidelines on diabetes, pre-diabetes, and cardiovascular diseases developed in collaboration with the EASD. Eur Heart J. 2020;41:255-323.

20. Fukushima M, Suzuki H, Seino Y. Insulin secretion capacity in the development from normal glucose tolerance to type 2 diabetes. Diabetes Res Clin Pract. 2004;66(Suppl 1):S37-43.

21. Action to Control Cardiovascular Risk in Diabetes Study Group, Gerstein HC, Miller ME, Byington RP, et al. Effects of intensive glucose lowering in type 2 diabetes. N Engl J Med. 2008;358:2545-59.

22. ADVANCE Collaborative Group, Patel A, MacMahon S, Chalmers J, et al. Intensive blood glucose control and vascular outcomes in patients with type 2 diabetes. N Engl J Med. 2008;358:2560-72.

23. Sone $H$, Tanaka $S$, Tanaka $S$, et al. Japan Diabetes Complications Study Group. Serum level of triglycerides is a potent risk factor comparable to LDL cholesterol for coronary heart disease in Japanese patients with type 2 diabetes: subanalysis of the Japan Diabetes Complications Study (JDCS). J Clin Endocrinol Metab. 2011;96:3448-56.

24. Kadowaki T, Inagaki N, Kondo K, et al. Efficacy and safety of canagliflozin as add-on therapy to teneligliptin in Japanese patients with type 2 diabetes mellitus: results of a 24-week, randomized, doubleblind, placebo-controlled trial. Diabetes Obes Metab. 2017;19:874-82.

25. Ueno $H$, Nakazato $H$, Ebihara E, et al. Effects of Ipragliflozin on postprandial glucose metabolism and gut peptides in type 2 diabetes: a pilot study. Diabetes Ther. 2018;9:403-11.

26. Osonoi T, Tamasawa A, Osonoi Y, Ofuchi K, Katoh M, Saito M. Canagliflozin increases postprandial total glucagon-like peptide 1 levels in the absence of $\alpha$-Glucosidase inhibitor therapy in patients with type 2 diabetes: a single-arm, non-randomized. Open-Label Study Diabetes Ther. 2019;10:2045-59.

27. Bertoni AG, Hundley WG, Massing MW, Bonds DE, Burke GL, Goff DC Jr. Heart failure prevalence, incidence, and mortality in the elderly with diabetes. Diabetes Care. 2004;27:699-703.

28. McGuire DK, Van de Werf F, Armstrong PW, et al. Trial Evaluating Cardiovascular Outcomes With Sitagliptin (TECOS) Study Group. Association between Sitagliptin use and heart failure hospitalization and related outcomes in type 2 diabetes mellitus: secondary analysis of a randomized clinical trial. JAMA Cardiol. 2016;1:126-35.

29. Balijepalli C, Shirali R, Kandaswamy P, et al. Cardiovascular safety of empagliflozin versus dipeptidyl peptidase-4 (DPP-4) inhibitors in type 2 diabetes: systematic literature review and indirect comparisons. Diabetes Ther. 2018;9:1491-500. 